\title{
Postface
}

\section{Quelques réflexions sur les milieux étudiants dans les dynamiques de démobilisation}

Some thoughts on student circles in demobilization dynamics

\section{Olivier Fillieule}

\section{(2) OpenEdition}

\section{Journals}

Édition électronique

URL : http://journals.openedition.org/ejts/4834

DOI : $10.4000 /$ ejts.4834

ISSN : $1773-0546$

Éditeur

EJTS

Référence électronique

Olivier Fillieule, "Quelques réflexions sur les milieux étudiants dans les dynamiques de

démobilisation », European Journal of Turkish Studies [En ligne], 17 | 2013, mis en ligne le 01 avril 2014, consulté le 16 février 2020. URL : http://journals.openedition.org/ejts/4834 ; DOI : 10.4000/ejts.4834

Ce document a été généré automatiquement le 16 février 2020.

(c) Some rights reserved / Creative Commons license 


\title{
Postface
}

\section{Quelques réflexions sur les milieux étudiants dans les dynamiques de démobilisation}

\author{
Some thoughts on student circles in demobilization dynamics
}

\author{
Olivier Fillieule
}

1 La place des étudiant.e.s dans la dynamique des luttes sociales n'a fait que croître au cours $\mathrm{du} \mathrm{xx}^{\mathrm{e}}$ siècle, en lien avec le développement des études secondaires et universitaires à partir des années soixante dans de nombreux pays du monde développé ou en développement. Dans la période la plus récente, comme le suggère d'entrée de jeu Jordi Tejel Gorgas dans l'introduction au dossier que l'on vient de lire, il est frappant de constater que les étudiant.e.s sont bien souvent aux avant-postes des mouvements de masse qui ont bouleversé la donne politique en Tunisie, en Égypte puis dans de nombreux États de la région, jusqu'au mouvement suscité par le projet de transformation du parc Gezi à Istanbul, à première vue porté par une fraction de la classe moyenne jeune, éduquée et laïque. Plus généralement, peu de mouvements révolutionnaires des 60 dernières années ne sont pas marqués par l'engagement décisif de ce groupe. Que l'on songe à la révolution hongroise de 1956 qui débute par des mobilisations étudiantes, aux manifestations étudiantes de 1960 en Corée du Sud qui font tomber le régime (et en Turquie avec moins de succès), à la première phase de la révolution culturelle chinoise en 1966-1968, aux nombreux mouvements de Mai 1968, à la Grèce de 1973, à l'Iran de 1978 et bien sûr au soulèvement des étudiants chinois en 1989. C'est dès lors très logiquement que la littérature historique et de sociologie politique sur les mobilisations étudiantes se révèle très riche.

2 Il n'est pas certain pourtant que toutes les dimensions propres aux mobilisations de ce groupe social aient été entièrement explorées. Autour notamment de trois éléments. D'une part, dans la mesure ou la sociologie des mobilisations est peu articulée aux travaux sur la socialisation ${ }^{1}$, et donc aux recherches sur les effets d'âge et de génération, il manque une exploration systématique des effets sur l'engagement de 
l'âge biologique comme de l'âge social des individus dans cette période de l'existence marquée par le phénomène des 'années impressionnables' relevé naguère par Mannheim (1928) et plus généralement par de multiples transitions dans toutes les sphères de l'existence (Fillieule 2013a et b) D'autre part, si les mouvements étudiants sont assez largement explorés per se, la littérature est plus rare sur la manière dont les étudiant.e.s s'inscrivent dans les milieux militants et dans les luttes qui dépassent les seules revendications étudiantes. Par exemple, la coexistence de cohortes d'âge dans les mouvements sociaux et ses effets, les rapports entre les étudiant.e.s et les autres membres de leurs classes d'âge n'occupant pas la même position structurale. Ce qui revient à explorer tout ce qui dans le statut d'étudiant.e favorise ou défavorise l'engagement politique. Ici la prise en considération de données macro sociologiques contextuelles ou culturelles fait parfois défaut, les auteur.e.s se contentant souvent d'invoquer la notion de 'disponibilité biographique'. Enfin, le devenir politique des étudiant.e.s, ce qu'ils ou elles deviennent et ce qu'ils ou elles font une fois sorti.e.s des études est surtout étudié au niveau micro sociologique des expériences biographiques des individus, aux dépens d'une réflexion plus large sur les cycles de mobilisation, sur la contribution d'unités de générations forgées dans les luttes d'étudiant.e.s à d'autres mouvements subséquents soit pour les initier soit pour les nourrir. C'est notamment sur ce dernier point que ce dossier de l'EJTS se révèle précieux. En prenant le parti de centrer le propos sur le devenir militant des étudiant.e.s politisé.e.s, dans une perspective comparée et autour des questions liées de la démobilisation, du désengagement et plus généralement des reconversions, c'est bien la question des mondes étudiant.e.s comme vivier de militant.e.s qui est posée.

Dans ce qui suit, sans prétendre opérer une synthèse des résultats offerts par ce riche dossier dont l'article introductif résume parfaitement les enjeux, je me contenterai de poursuivre quelque peu le débat en avançant quelques remarques autour des mobilisations étudiantes et des logiques de la démobilisation et du désengagement.

\section{Effets d'âge et de génération. 'The elephant in the room'}

Dans un article consacré à la question de l'importance des cohortes d'âge au sein des mouvements, Hank Johnston (2012) souligne un paradoxe : les facteurs générationnels sont aussi visibles qu'un éléphant dans une pièce mais ils ne sont que très rarement au cœur des recherches sur les mobilisations sociales et politiques. Pourtant celles-ci sont bien souvent autant le fruit de phénomènes générationnels qu'un facteur de la construction de ce phénomène. De ce point de vue, le mouvement des droits civiques, le mouvement féministe, le mouvement étudiant des années 1960' aux USA, le mouvement de Mai 1968 et les luttes qui en découlent dans la décennie suivante sont tous fortement marqués par des facteurs générationnels dont la prise en compte permet de mieux saisir à la fois leur émergence, leurs développements, via les effets produits par la succession et la coexistence de micro-cohortes (Whittier 1995 ; Fillieule, Blanchard 2013), et leurs conséquences, tant sur le plan individuel des conséquences biographiques, sur le plan organisationnel de la diffusion et des transferts de savoirfaire, de capital relationnel et de constructions idéologiques que sur le plan des changements macro sociaux à plus long terme dans les valeurs, les représentations sociales et les conduites (Goldstone, McAdam 2001). En centrant l'attention sur les 
mouvements étudiant.e.s et le devenir de leurs membres, le présent dossier appelle à mettre ces questions au cœur du dispositif d'enquête.

En premier lieu, en se demandant par quel processus l'exposition d'individus à une 'dynamique de déstabilisation' peut générer un 'ensemble générationnel' au sens de Karl Mannheim (1928), ou dit autrement, comment 'le temps court parvient à accoucher d'un temps long' (Ihl 2002: 125), tout particulièrement lorsque ce temps court est marqué par des épreuves particulièrement douloureuses et marquantes du fait de la répression subie (interruption forcée des études, clandestinité, emprisonnement, torture, etc). Ainsi Élise Massicard (2013) montre-t-elle, à propos des entrepreneurs politiques du mouvement aléviste, comment pour la génération en âge de poursuivre des études supérieures à la fin des années 1970, la répression a signifié l'interruption des cursus et l'impossibilité d'atteindre dans les décennies suivantes des professions valorisées et des positions de responsabilité dans les organisations alévistes, alors que pour la génération suivante, le coup d'État de 1980 ne vient pas interrompre des études supérieures, lesquelles détermineront pour des militant.e.s issu.e.s le plus souvent de milieux modeste, une ascension sociale en même temps que des engagements à haut niveau de responsabilité. De son côté, Kostis Kornetis distingue de la même manière deux générations dans le mouvement étudiant grec des années soixante. La première voyant ses destins sociaux et son champ des possibles totalement modelé par l'expérience de la dictature alors que la génération suivante n'était qu'au tout début de l'adolescence :

'Those teenagers would come of age under the regime and for that reason would better understand its logic, exploiting the political opportunities that the dictatorship unwillingly allowed, in order to develop political disruption and everyday creativity. By that time, people of the first cohort, who had experienced the toughest years of the junta's authority, restrictions, martial law and full-blown preventive censorship, and who had been more prone to clandestine networks and armed resistance, were already imprisoned or exiled.' (2013: 84)

Kostis Kornetis suggère que pour la première génération, l'échec est tellement cuisant que ses membres vont développer une très grande méfiance à l'égard de la résurgence de l'action de masse, sombrant dans une sorte de mélancolie après avoir passé leur vingtaine en prison. Du coup ils ne soutiennent pas ou fort peu le mouvement étudiant de 1973. À l'inverse, pour la génération suivante qui sera au cœur du mouvement de 1973, la lutte armée et la clandestinité apparaissent comme des modes d'action totalement dépassés, inefficaces, voire ringards :

'In Greece by the end of 1972, and with a mass student movement that generated an entirely different repertoire of action, it was clear that 'vanguard organizations' were becoming increasingly obsolete.' (Kornetis 2013:87)

7 En second lieu, en s'interrogeant sur la spécificité des mécanismes socialisateurs propres aux jeunes adultes. Le modèle longtemps dominant dit de la persistance, pour lequel les apprentissages 'préadultes' perdurent tout au long de la vie, et se renforcent avec le temps, avec pour effet de rapporter les attitudes politiques à de simples effets d'âge sans prendre en compte les effets de cohorte, de cycle de vie et de période, est depuis longtemps battu en brèche (Searing, Schwartz, Lind 1973; Jennings, Niemi 1981). Cela n'a pas pour autant débouché sur une exploration très poussée des modèles alternatifs, comme celui du cycle de vie ouvert (lifelong openness model), qui met l'accent sur les possibilités de changement tout au long de l'existence (Sigel 1989; Sapiro 1994) et qui de ce fait est très proche d'un autre modèle, celui des années impressionnables, 
dans lequel les préférences et attitudes individuelles continuent à se cristalliser entre la fin de l'adolescence et le début de la vie adulte, après quoi elles sont relativement stabilisées (Sears, Levy 2003). Ce modèle est directement inspiré d'un ouvrage important de Karl Mannheim, Le problème des générations (1928), lequel suggère que les années durant lesquelles se constitue un horizon politique personnel et réflexif commencent autour de 17 ans : l'individu aménage alors consciemment son 'tréfonds psychique', autrement dit remet en cause ses dispositions au profit d'une 'nouvelle orientation' imposée par la configuration socio-historique dans laquelle il s'inscrit. Pour Karl Mannheim c'est donc au cours de cet âge (qu'il fixe de manière sans doute trop rigide entre 17 et 25 ans) que les individus recomposent leurs apprentissages primaires et 'fixent' leur rapport au monde. Cette analyse pose les jalons d'un questionnement approfondi sur les processus concrets qui nourrissent cette phase de la construction individuelle et, par extension, la dynamique des générations. Plus encore, en forgeant la notion d'unité de génération, Karl Mannheim souligne combien les individus appartenant à une même génération politique, même s'ils partagent un certain nombre de caractéristiques, lesquelles expliquent en partie leur ralliement à un moment donné à une cause et une organisation, connaissent cependant des degrés variés de proximité les un.e.s aux autres, en raison de leurs dispositions et positions et, partant, de leurs intérêts, de leurs motivations à s'engager, de leur perception des enjeux de la lutte et, finalement de la manière dont ils endossent telles ou telles identités collectives. Ce dernier point suggère, ce que les auteur.e.s du présent dossier font de manière parfois trop rapide, de bien distinguer d'un côté ce qui contribue à faire des étudiant.e.s dans tel ou tel contexte un groupe unifié (un statut et des activités partagés, la fréquentation d'espaces concrets et symboliques communs, une distance objective et subjective aux autres personnes appartenant à leurs classes d'âge, ce tout particulièrement dans des situations où l'accès aux études est réservé à une petite élite) et de l'autre tout ce qui au sein de ces groupes contribue à distinguer différentes unités, en fonction des positions des un.e.s ou des autres dans les rapports de domination liés à l'âge, à la classe, au sexe et à la race. Kostis Kornetis montre par exemple très bien dans son ouvrage consacré au mouvement étudiant grec des années 1960 comment la massification de l'enseignement, l'absence corrélative de perspectives professionnelles pour les nouveaux diplômés dans un pays peu développé, plutôt que de contribuer à constituer un groupe homogène lié par une communauté de destin, exacerbe au contraire les écarts, en faisant coexister des héritiers et des transfuges de classe, des individus dont les diplômes ne sont que la sanction d'un statut social et de chances objectives déjà acquis et d'autres pour lesquels le diplôme est l'unique voie d'ascension sociale possible. Kostis Kornetis conclut que la position de classe

'could be a major deterrent to activism, because risking a university degree was a dangerous possibility for people from the lower classes. [...] To this one could add the relative social isolation that students with solid working-class backgrounds might experience at the universities.' (Kornetis 2013: 108) ${ }^{2}$

En troisième lieu, signalons une dernière piste de recherche, qui s'appuie sur les progrès récents des sciences cognitives et de la psychologie du développement pour tenter de reposer à nouveaux frais les éléments propres aux comportements des jeunes adultes et donc des années impressionnables comme étape distincte du développement (Arnett 2000). À ce propos Hank Johnston (2013) distingue quatre résultats: Premièrement, l'idée qu'il existe des schémas cognitifs spécifiques à la manière dont les 18-25 ans affrontent les situations d'incertitude. Les jeunes adultes chercheraient des 
stratégies innovantes alors que les plus âgés, face à l'inattendu, se replieraient plus volontiers sur les routines apprises, les expériences passées (Blanchard-Fields, Kalinauskas 2009: 3). Remarque qui n'est pas sans intérêt pour qui cherche à comprendre comment les acteurs calculent en situation de fluidité politique, lorsque les échanges de coup tendent à ne plus fonctionner selon une logique ordinaire. Ce que suggèrent ces travaux, c'est que plutôt que d'une 'évasion des calculs' (Dobry 1986) généralisée et touchant tous les acteurs au même titre, sans doute que les individus les plus jeunes, dont on a souligné plus haut l'importance dans tout un ensemble de crise politique, sont moins soumis.e.s aux 'anticipations mutuelles' (Goffman 1969: 85-145) et aux 'processus d'alignement' (Ermakoff 2008) que leurs aîné.e.s, ouvrant potentiellement une voie à une meilleure compréhension des micro-fondations de l'action en situation de crise et aux mécanismes d'agrégation en situation d'incertitude. Deuxièmement, le constat que les jeunes adultes sont plus prompts à s'engager dans des conduites à risque que leurs aînés, ayant moins à disposition d'expériences permettant d'évaluer les risques et les coûts (Blanchard-Fields 2007 ; Blanchard-Fields, Mienaltowski, Seay 2007). Troisièmement, la capacité régulatrice des émotions serait positivement associée avec l'âge chronologique (Carstensen, Mikels, Mather 2006; Mather 2010). Dès lors en situation de mobilisation, les actions spontanées suscitées par l'émotion seraient plus fréquentes que chez des personnes plus âgées. Donc la densité des émotions décroîtrait avec l'âge. Enfin, les mécanismes de déclenchement cognitifs (ce que Hank Johnston nomme le cognitive triggering), soit le fait de rompre avec les schémas appris du passé et de voir des opportunités et des possibilités d'action nouvelles en situation, serait un des traits marquant des mobilisations étudiantes :

'It partly accounts for the general phenomenon of student unrest and mobilization, for they see things in ways their elders do not. Interpretative schemas of 18 to 25 yo are less encumbered by past experiences; which allows for greater processing speed and the ability to make connections with other schemas more quickly.' (Johnston 2013: 50)

D'une certaine manière tous ces résultats nous ramènent aux intuitions de Mannheim, notamment autour de l'idée des années impressionnables comme succession de 'fresh encounters' avec des situations inédites poussant à l'invention et l'accumulation rapide d'expériences et fait aussi écho aux travaux de Kenneth Keniston (1968). Il reste que pour l'heure les recherches dans le domaine demeurent fragiles dans leurs résultats et les techniques liées à l'imagerie cérébrale auquel il est le plus souvent fait recours est encore loin d'offrir des protocoles de recherches entièrement convaincants et suffisamment solides pour autoriser de telles généralisations à propos des logiques de l'action protestataire.

\section{Démobilisation et désengagement}

10 On ne sait pas grand-chose sur les mécanismes qui président au déclin des mouvements sociaux et aux formes variées de démobilisation individuelle ou collective qui génèrent ce déclin ou le soldent (Taylor 1989: 772 ; voir aussi Fillieule 2005, 2010)3. Pourtant, la contrepartie logique des processus de mobilisation initiale et de recrutement est bien la démobilisation collective et le désengagement individuel.

11 La notion de démobilisation renvoie à une pluralité de phénomènes allant du désengagement individuel à la démobilisation politique d'une société toute entière, la 
somme des comportements individuels produisant des cycles macro sociaux d'engagement ou de retrait sur la sphère privée (Hirschman : 1983). Plus précisément, au-delà des cycles d'intérêt pour la politique, l'on peut distinguer analytiquement quatre autres types de situation. Premièrement les phénomènes de démobilisation d'un champ multi organisationnel (Curtis, Zurcher 1973), c'est-à-dire d'un secteur entier de lutte, avec ses organisations formelles ses réseaux de soutien et ses compagnons de route. Les articles de ce dossier en donnent un certain nombre d'illustrations. Deuxièmement, la démobilisation peut porter moins sur un secteur que sur une campagne de mobilisation lorsque celle-ci s'essouffle, du fait de sa réussite ou de son échec, associée parfois à un désinvestissement de tel ou tel lieux concret de lutte au profit d'autres. Au niveau mesosociologique, la démobilisation peut frapper une organisation spécifique de mouvement social, soit par autodissolution, soit du fait d'un déclin général d'une cause soit encore sous l'effet de la répression (interdiction, décapitation du leadership) comme l'attestent plusieurs des études de cas réunies ici. $\mathrm{Au}$ niveau micro sociologique enfin, le désengagement individuel peut s'inscrire dans tous ces cas de figure mais aussi renvoyer à des trajectoires idiosyncrasiques. De ce point de vue, la démobilisation individuelle n'est pas toujours volontaire. Elle peut aussi bien résulter de l'auto dissolution d'un collectif, du déclin d'une idéologie, comme l'illustre Verta Taylor (1989) à propos du féminisme américain d'après-guerre, d'une exclusion, d'une déprogrammation, soit encore d'une mise hors-jeu par l'exil forcé, une peine de prison.

Les modalités de la défection individuelle, aussi bien, sont variables. Celle-ci peut être isolée ou collective, à l'occasion par exemple d'une scission, ou lorsque les départs se font dans une logique de groupes affinitaires. Massimo Introvigne distingue les defectors, qui partent de manière négociée, les apostats, qui deviennent des ennemis professionnels de leur organisation, les partants ordinaires, enfin, qui disparaissent sans qu'apparemment leur désengagement ne représente un coût sensible, pour eux ou pour l'organisation (1999). Typologie qu'il faut compléter par toutes les formes de défection passive et de mise en retrait (Fillieule, Bennani-Chraïbi 2003), mais aussi par tous les cas dans lesquels le désengagement est suivi, et quelquefois provoqué, par l'entrée dans une autre organisation.

Tout se complique cependant dès lors que l'on envisage tout un ensemble de situations pouvant s'analyser en terme de désengagement, mais de 'désengagement dans la fidélité's. Sans développer ce point complexe, mentionnons à titre d'exemple la poursuite des engagements militants dans une direction réformiste, particulièrement fréquente dans les cas des activistes des mouvements étudiants arrivés en fin d'études ou encore subissant une répression accrue. Dina El Khawaga l'a bien montré dans son analyse du devenir des jeunes militant.e.s des mouvements de 1968 puis de 1972 en Égypte. À la fin des années 1970, trois facteurs favorisent l'abandon des stratégies confrontatives: la dureté de la répression des mouvements de 1968 et de 1972 ; la normalisation des rapports avec Israël et l'essai de démocratisation par le haut; la fin des études enfin, qui implique l'entrée sur le marché du travail. C'est dans un premier temps 'de l'intérieur' que ces militant.e.s vont essayer de continuer la lutte, en participant aux 'comités spécialisés' des partis et dans la recherche de tribunes libres alternatives, à travers le métier de journaliste ou d'avocat, autant de professions qui permettent de ne rien renier de ses convictions militantes tout en assurant un reclassement professionnel. Les limites de la démocratisation partisane se font cependant vite sentir et c'est alors sur le militantisme au sein des organisations 
professionnelles que se concentrent ces militant.e.s: 'les ordres professionnels, les syndicats d'avocats, de journalistes, et dans une moindre mesure d'ingénieurs et de médecins'. L'analyse permet ici d'articuler les changements dans la sphère des engagements publics avec les changements dans la carrière professionnelle (sortie des études et entrée dans la vie active), en rapportant les conditions de possibilité du militantisme comme de la défection à la possible variation des opportunités professionnelles, tout particulièrement dans les configurations où les ressources disponibles peuvent faire l'objet d'une reconversion dans le champ des activités salariées ${ }^{5}$.

Pour des raisons évidentes liées à la nécessaire entrée sur le marché du travail à un moment donné du parcours, l'articulation entre pratiques professionnelles et pratiques d'engagement est particulièrement intéressante à explorer lorsque l'on travaille sur le devenir des étudiant.e.s contestataires. Ce notamment dans trois directions : en se centrant sur la politisation des pratiques professionnelles (e.g. Muel-Dreyfus, 1983; Israël, 2005 ; Lechaux, Roussel, 2010), la reconversion de ressources acquises dans le militantisme dans la sphère des activités professionnelles (e.g. Ollitrault 2008; Broqua 2005 ; Tissot et al. 2006; Voegtli 2009), l'orientation des choix de carrière en fonction des convictions idéologiques et des expériences militantes (e.g. Fendrich 1993 ; Whalen, Flacks 1989; McAdam 1989; Dauvin, Siméant 2002; Willemez 2003; Pagis 2009; Champy, Israël 2009 ; Neveu 2008), sans oublier les riches travaux sur le cause lawyering (dont on trouvera une présentation dans Agrikoliansky 2010).

$\mathrm{Si}$, on le voit bien à la profusion des références, l'articulation entre sphères de vie militante et professionnelle est assez largement explorée, il est plus rare de trouver des travaux qui en même temps s'intéressent à une troisième sphère de vie, celle des relations affectives et familiales. Et pourtant, si l'on se penche sur le devenir des activistes dans les mouvements étudiants, il apparaît bien difficile de négliger cette dernière dimension, les contraintes de l'entrée sur le marché du travail et les aspirations à remplir les obligations de 'l'ethos familial' étant assez largement consubstantielles. Comme le souligne Patrick Haenni à propos des jeunes militants islamistes Égyptiens, "le "besoin de famille" auquel est exposé tout mouvement de jeunes [est] un facteur clé de la démobilisation [...] à partir de la fin des années 1980. En effet, après cinq ou six ans d'engagement, ils furent nombreux à se trouver placés devant l'échéance du mariage. L'engagement militant ne permettait que difficilement d'accéder au statut d'époux qui suppose une situation économique et sociale stable ainsi qu'une bonne image de moralité dans le quartier. Or, depuis la fixation du mouvement sur le pourchas du mal, cela cadrait mal avec l'engagement militant. Chez beaucoup, celui-ci céda devant le désir d'insertion sociale et économique. L'invocation du désaveu de la violence "islamiste" servira alors d'argument à leur sortie de militance qui est surtout un transfert d'allégeance vers des réseaux plus porteurs d'opportunités par rapport aux demandes d'insertion sociale et professionnelle des jeunes' (2001: 204).

Si la recherche a accompli d'indéniables progrès dans la compréhension des mécanismes de la démobilisation individuelle, et le présent dossier en est un nouveau témoignage, un certain nombre de pistes demeurent insuffisamment explorées. Ainsi par exemple, au niveau mesosociologique, nous manquons encore d'une typologie bien 
assurée de la diversité des trajectoires suivies par les organisations de mouvement social qui connaissent un processus de démobilisation. Certains cas de figure sont bien identifiés, à commencer par les trajectoires de bureaucratisation, d'institutionnalisation et d'assimilation par l'État, ou à l'inverse de radicalisation, à quoi on ajoutera encore les périodes d'abeyance, mises en lumière par Leila J. Rupp et Verta Taylor (1987) et parfaitement illustrées par Maryjane Osa (2003), mais cela demeure encore insuffisant. Aussi bien, la recherche pourrait plus systématiquement étudier la manière dont tels ou tels contextes macrosociaux freinent ou favorisent telles ou telles trajectoires de démobilisation. L'existence ou la mise à disposition (le plus souvent par l'État) de possibilités de reconversion en est un exemple. De ce point de vue, la littérature sur les mouvements dits terroristes ou sur les sorties de conflits armés, autour notamment de la question des repentis et des programmes de réhabilitation, mais aussi des politiques publiques de sortie de crise pour favoriser le désarmement des groupes armés constitue un outil précieux pour réfléchir à ce qui freine ou accélère les phénomènes de démobilisation au niveau meso comme microsociologique (Björgo and Horgan, 2009 ; Sommier 2012 ; Fillieule 2013c).

Enfin, les conséquences à court et moyen terme des phénomènes de démobilisation politique posent aussi une série de questions passionnantes auxquelles pour l'instant la littérature a accordé peu d'attention, si ce n'est sur le plan des conséquences biographiques de l'engagement. Ainsi par exemple, et pour finir, si l'on s'interroge beaucoup sur la diffusion des mouvements sociaux dans une perspective positive, il serait intéressant aussi de poser la question des effets dans le temps et dans l'espace de l'échec d'un mouvement, d'une campagne ou d'une organisation sur d'autres campagnes ou mouvements, que ces derniers s'inscrivent dans un champ d'alliance ou un champ de conflit. L'on accèderait ainsi à une meilleure compréhension des conséquences de l'écrasement de la Commune de Paris en 1871, des révolutions de 1848, du mouvement étudiant chinois en 1989 et, plus récemment, de l'échec pathétique de la révolution égyptienne de ce siècle.

\section{BIBLIOGRAPHIE}

Agrikoliansky, E. (2010) 'Les usages protestataires du droit’, in Fillieule O.; Agrikoliansky E., Sommier I., Penser les mouvements sociaux, Paris, La Découverte.

Arnett, J. J. (2000) 'Emerging Adulthood: A theory of development from the late teens through the twenties', American psychologist (55): 469-480. URL: http://www.jeffreyarnett.com/articles/ ARNETT_Emerging_Adulthood_theory.pdf (accès 15 décembre 2013).

Björgo, T.; Horgan, J. (eds) (2009) Leaving Terrorism Behind: Individual and collective disengagement, Londres / New York, Routledge.

Blanchard-Fields, F. (2007) ‘Everyday problem solving and emotion: An adult developmental perspective. Current Directions in Psychological Science' 16: 26-31. URI: http://dx.doi.org/ 10.1111/j.1467-8721.2007.00469.x (accès 15 décembre 2013). 
Blanchard-Fields, F.; Seay, R. B.; Mienaltowski, A. (2007) 'Age differences in everyday problemsolving effectiveness: Older adults select more effective strategies for interpersonal problems', Journals of Gerontology: Psychological Sciences 62: 61-64. URL: http:// psychsocgerontology.oxfordjournals.org/content/62/1/P61

Broqua C. (2005) Agir pour ne pas mourir, Paris, Presses de science po.

Carstensen, L. L.; Mikels, J. A.; Mather, M. (2006) 'Aging and the intersection of cognition, motivation and emotion', in Birren, J.; Schaie, K. W. (eds.), Handbook of the Psychology of Aging, Burlington, MA / San Diego, CA, Academic Press, pp. 343-362.

Champy F. ; Israël L. (2009) 'Professions et engagement public', Sociétés contemporaines 73(1) : 7-19. URI: http://dx.doi.org/10.3917/soco.073.0007 (accès 15 décembre 2013).

Collovald, A. ; Willemez, L.,=; Rozier, S.; Lechien, M.-H. (2002) L'humanitaire ou le management des dévouements. Enquête sur un militantisme de "solidarité internationale" en faveur du Tiers-Monde, Rennes, Presses universitaires de Rennes.

Curtis R. L., Zurcher L. A. (1973) ‘Stable Resources of Protest Movements: The MultiOrganizational Field', Social Forces, 52 (1): 53-51. URL: http://www.jstor.org/stable/2576423 (accès 15 décembre 2013).

Dauvin P., Siméant J. (2002) Le travail humanitaire. Les acteurs des ONG du siège au terrain, Paris, Presses de Sciences Po.

Devaux, S. (2005) Engagements associatifs et postcommunisme. Le cas de la République tchèque, Paris, Belin.

Dobry, M. (1986) Sociologie des crises politiques, Presses de Sciences Po, Paris.

El Khawagha, D. (2003) 'La génération seventies en Égypte. La société civile comme répertoire d'action alternatif', pp. 271-292 in Bennani-Chraibi M., Fillieule O. (eds), Résistances et protestations dans les sociétés musulmanes, Paris, Presses de Sciences Po. URL: http://www.cairn.info/ article.php?ID_ARTICLE=SCPO_BENNA_2003_01_0271 (accès 15 décembre 2013).

Ermakoff, I. (2008) Ruling Oneself Out. A Theory of Collective Abdications, Durham, Duke University Press.

Fendrich, J. M. (1993) Ideal Citizens, Albany, NY: State University of New York Press.

Fillieule, O. (2013a) 'Political Socialization and social movements', in Snow, D.; Klandermans, B.; Della Porta, D.; McAdam, D. (eds), The Wiley-Blackwell Encyclopedia of Social and political Movements. URI: http://dx.doi.org/10.1002/9780470674871.wbespm199

Fillieule, O. (2013b) 'Age and social movements', in Snow, D.; Klandermans, B.; Della Porta, D.; McAdam, D. (eds) The Wiley-Blackwell Encyclopedia of Social and political Movements. URI: http:// dx.doi.org/10.1002/9780470674871.wbespm004

Fillieule, O. (2013c) 'Le désengagement d'organisations radicales. Approche par les processus et les configurations', Lien social et politiques, $\mathrm{n}^{\circ}$ 68, 2012 : 37-59. URI: http://dx.doi.org/ 10.7202/1014804ar

Fillieule, O. (Ed.) (2005) Le Désengagement militant (Paris : Belin).

Fillieule, O., (2010) 'Some Elements of an Interactionist Approach to Political Disengagement', Social movement studies, vol 9, $\mathrm{n}^{\circ}$ 1: 1-15. URI: http://dx.doi.org/10.1080/14742830903442436

Fillieule, O. ; Bennani-Chraïbi M., (2003) 'Exit, voice, loyalty et bien d'autres choses encore...', in Bennani-Chraibi M.; Fillieule O. (eds), Résistances et protestations dans les sociétés musulmanes, Paris, 
Presses de Sciences Po, pp. 43-126. URL: http://www.cairn.info/article.php?

ID_ARTICLE=SCPO_BENNA_2003_01_0043 (accès 15 décembre 2013).

Fillieule, O.; Blanchard, P. (2013) 'Fighting Together : Assessing Continuity and Change in Social Movement Organisations through the Study of Constituencies' Heterogeneity', in Kauppi, N. (ed.), A Political Sociology of transnational Europe, Colchester, ECPR Press, pp. 79-11.

Goffman, E. (1969) Strategic Interaction, Philadelphie, University of Pennsylvania Press.

Goldstone, J.; McAdam, D. (2001) 'Contention in Demographic and Life-Course Context', in Aminzade, R.; Goldstone, J.; McAdam, D.; Perry, E. (eds), Silence and Voice in the Study of Contentious Politics, Cambridge, Cambridge University Press, pp. 195-221. URI: http://dx.doi.org/10.1017/ CB09780511815331.009 (accès 15 décembre 2013).

Grojean, O. (2013) 'Comment gérer une crise politique interne ? Façonnage organisationnel du militantisme, maintien de l'engagement et trajectoires de défection', Politix, 2 (102) : 63-88. URI: http://dx.doi.org/10.3917/pox.102.0063 (accès 15 décembre 2013).

Haenni, P. (2001) 'Banlieues indociles. Sur la politisation des quartiers périphériques du Caire’, thèse de doctorat, Institut d'études politiques, Paris.

Hirschman, A. O. (1983) Bonheur privé, action publique, Paris, Fayard.

Ihl, O. (2002) ‘Socialisation et événements politiques’, Revue française de science politique, 52 (2) : 125-144. URI: http://dx.doi.org/10.3406/rfsp.2002.403704 (accès 15 décembre 2013).

Introvigne, M. (1999) 'Defectors, ordinary leavetakers and apostates: a quantitative study of former members of New Acropolis in France', Nova Religio, The Journal of Alternative and Emergent Religions 3(1): 83-99. URL: http://www.jstor.org/stable/10.1525/nr.1999.3.1.83 (accès 15 décembre 2013).

Israël, L. (2005) Robes noires, années sombres. Avocats et magistrats en résistance pendant la Seconde guerre mondiale, Paris, Fayard.

Jennings, M. K.; Niemi, R. G. (1981) Generations and Politics: A panel study of young aduls and their parents, Princeton, Princeton University Press.

Johnston, H. (2012) 'Age Cohorts, Cognition and Collective Violence', in Seferiades S.; Johnston, H. (eds), Violent Protest, Contentious Politics, and the Neoliberal State, Edited by S. Farnham / Burlington, Ashgate, pp. 39-51.

Keniston, K. (1968), Young Radicals, New York, Harcourt and Brace / Jovanovich.

Kornetis, K. (2013) Children of the Dictatorship, Student Resistance, Cultural Politics, and the 'Long 1960s' in Greece, New York / Oxford, Berghahn Books.

Lechaux, B.; Roussel V. (2010) Voicing Dissent: American Artists and the War on Iraq, Londres, Routledge.

Mannheim, K. (1952) 'The Problem of Generations,' in Essays on the Sociology of Knowledge, Londres, Routledge and Kegan Paul, pp. 277-332. URL: https://archive.org/details/ essaysonsociolog00mann (accès 15 décembre 2013).

Massicard, É. (2013) 'Quand le militantisme s'adapte au terrain. Continuités et discontinuités dans les carrières militantes au sein du mouvement aléviste en Turquie et en Allemagne', Politix 102(2) : 89-108. URI: http://dx.doi.org/10.3917/pox.102.0089 (accès 15 décembre 2013).

Mather, M. (2010) 'Aging and cognition', Wiley Interdisciplinary Reviews: Cognitive Science 1(3): 346-362. URI: http://dx.doi.org/10.1002/wcs.64 (accès 15 décembre 2013). 
McAdam, D. (1989) 'The biographical consequences of activism', American Sociological Review 54: 744-760. URL: http://www.jstor.org/stable/2117751 (accès 15 décembre 2013).

Muel-Dreyfus, F. (1983) Le métier d'éducateur. Les instituteurs de 1900, les éducateurs spécialisés de 1968, Paris, Éditions de Minuit.

Neveu, E. (2008) ‘Trajectoires de “soixante-huitards” ordinaires’, in Damamme D.; Gobille, B.; Ollitrault, S. (eds), Militer pour la planète : sociologie des écologistes, Rennes, Presses universitaires de Rennes.

Osa, M. (2003) Solidarity and Contention: Networks of Polish Opposition, Minneapolis, University of Minnesota Press.

Pagis, J. (2009) 'Les incidences biographiques du militantisme en Mai 68. Une enquête sur deux générations familiales : des "soixante-huitards" et leurs enfants scolarisés dans deux écoles expérimentales', thèse de doctorat de sciences sociales, ENS / EHESS.

Raison du Cleuziou, Y. (2010) 'Des fidélités paradoxales. Recomposition des appartenances et militantisme institutionnel dans une institution en crise’, in Lagroye, J.; Offerlé, M. (eds), Sociologie de l'institution, Paris, Belin.

Rupp, L.; Taylor, V. (1987) Survival in the Doldrums, New York / Oxford, Oxford University Press. Sandell. R. (1999) 'Organizational life Aboard the Moving Bandwagons: A Network Analysis of dropouts from a Swedish Temperance Organization, 1896-1937', Acta Sociologica 42: 3-15. URL: http://www.jstor.org/stable/4201118 (accès 15 décembre 2013).

Sapiro, V. (1994) 'Political Socialization during Adulthood: Clarifying the Political Time of Our Lives', Research in Micropolitics 4: 197-223.

Searing, D.; Schwartz, J.; Lind, A. (1973) ‘The Structuring Principle: Political Socialization and Belief Systems', American Political Science Review, 67: 415-32.

Sears, D. O.; Levy, S. (2003) 'Childhood and Adult Political Development', in Sears, D. O.; Huddy, L.; Jervis, R. (eds), Oxford Handbook of Political Psychology, Oxford, Oxford University Press.

Sigel, R. (ed.) (1989) Political Learning in Adulthood, Chicago, University of Chicago Press.

Sommier, I. (2012) 'Engagement radical, désengagement et déradicalisation. Continuum et lignes de fracture', Lien social et politiques 68: 15-36. URI: http://dx.doi.org/10.7202/1014803ar (accès 15 décembre 2013).

Suaud, C. (1982) 'Conversions religieuses et reconversions économiques', Actes de la recherche en sciences sociales 44-45 : 72-94. URL: http://www.persee.fr/web/revues/home/prescript/article/ arss_0335-5322_1982_num_44_1_2169 (accès 15 décembre 2013).

Taylor. V., (1989) ‘Social Movement Continuity: The Women's Movement in Abeyance', American Sociological Review 54 (5): 761-775. URL: http://www.jstor.org/stable/2117752 (accès 15 décembre 2013).

Tissot, S., Gaubert, C., Lechien, M-H. (eds) (2005) Reconversions militantes, Limoges, Presses universitaires de Limoges.

van der Veen, G.; Klandermans, B (1989) “"Exit” Behavior in Social Movements Organizations', International Social Movement Research 2: 179 -198.

Voegtli, M. (2009) Luttes contre le sida, luttes homosexuelles : histoires croisées d'engagements militants en Suisse, thèse de science politique, UNIL / EHESS. 
Whalen, J.; Flacks, R. (1989) Beyond the Barricades: The Sixties Generation Grows Up, Philadelphie, Temple University Press).

Whittier, N. (1995) Feminist Generations. The persistence of the radical women's movement, Philadelphie, Temple University Press.

Willemez, L. (2003) 'Engagements professionnels et fidélités militantes. Les avocats travaillistes dans la défense judiciaire des salariés', Politix, vol 16, n 62, p. 145-164. URI: http://dx.doi.org/ 10.3406/polix.2003.1280

Zwerman, G.; Steinhoff, P. (2005) 'When Activists Ask for Trouble: State-Dissident Interactions and the New Left Cycle of Resistance in the United-States and Japan', in Davenport C.; Johnston H.; Mueller C. (eds), Repression and Mobilization, Minnesota: University of Minnesota Press.

\section{NOTES}

1. La socialisation politique renvoie à l'ensemble des mécanismes d'acquisition de valeurs politiques et d'attitudes face au vote et aux institutions représentatives. Elle peut ainsi être conçue comme l'acquisition d'une 'compétence politique' indexée sur un ensemble de savoirs légitimes. On en retient ici une acception plus extensive et dynamique en la concevant comme un processus relationnel et continu d'intériorisation de schèmes de perception et d'action relatifs au monde politique ou participant d'un rapport politique au monde social. Autrement dit, tous les éléments de la socialisation sont susceptibles de fonctionner comme des opérateurs d'identification et d'appréciation politiques dès lors qu'ils structurent le rapport des individus à eux/elles-mêmes, à leurs univers d'appartenance et au monde environnant. Cette définition repose sur trois éléments. En premier lieu, la socialisation politique est un processus socialement et historiquement déterminé, dépendant des systèmes d'appartenance des individus et des contextes dans lesquels ils s'inscrivent. Deuxièmement, la socialisation politique est un processus continu et dynamique au cours duquel les acquisitions enfantines et adolescentes (la 'socialisation primaire') sont aménagées ou transformées au cours du cycle de vie. Enfin, la dimension politique est constamment en jeu dans le processus de socialisation sans se résumer à un domaine de connaissances et d'activités spécialisées : elle renvoie à un système de schèmes cognitifs et pratiques reconnus ou non comme 'proprement politiques' mais ayant des effets de politisation (Fillieule 2013a).

2. Ailleurs dans son ouvrage, Kostis Kornetis souligne aussi la ligne de clivage entre étudiants issus de la campagne et citadins, cette différence recouvrant, mais partiellement, les différences de classe. Dans son analyse des ex soixante-huitards français, Julie Pagis (2009) a bien montré à quel point les variations dans les incidences biographiques de l'engagement étaient aussi redevable des formes de politisation antérieures à l'événement, au sexe, à l'origine sociale, à l'âge, etc.

3. À quelques rares exceptions bien entendu, parmi lesquelles notamment Sandell (1999); van der Veen, Klandermans (1989).

4. Nous empruntons le terme à Yann Raison du Cleuziou (2010: 748 et suivantes). Celui-ci pointe par là une forme de désaffiliation par reconversion des formes renouvelées de fidélité à la cause ou à l'institution 'historique', ici l'ordre dominicain. Voir également Olivier Grojean (2013) pour une même remarque à propos du maintien d'une mouvance PKK aux côtés de l'organisation au sens strict après le tournant historique opéré suite à l'arrestation d'Abdullah Öcalan.

5. Voir notamment, sur la question des reconversions: Suand (1982); Collovald et al. 2002 ;Bennani-Chraïbi, Fillieule 2003 ; Devaux 2005 ; Tissot, Gaubert, Lechien, 2006. 


\section{AUTEUR}

OLIVIER FILLIEULE

Université de Lausanne, Institut d'études politiques et internationales

Centre de recherche sur l'action politique de l'Université de Lausanne (CRAPUL) 